\title{
Monitoring of the Vegetation Index Distribution in the South and Southeast Asian Region with NOAA AVHRR Satellite Images
}

\author{
Suehiro Otoma, Yoshifumi Yasuoka, Tadakuni Miyazaki, Akira Shimizu and \\ Yasumi Fujinuma \\ National Institute for Environmental Studies \\ (Onogawa 16-2, Tsukuba, Ibaraki 305, Japan)
}

\begin{abstract}
Mosaiced cloud-free images and distributions of the vegetation index for 1986, 1987, 1988, 1987/1988, 1989, 1993 and 1994 were produced from NOAA AVHRR Local Area Coverage (LAC) satellite data in order to monitor the vegetation and land cover conditions in the Southeast Asian region on a macroscopic scale. These images were obtained by applying precise radiometric and geometric corrections which maintained the original $1 . \mathrm{km}$ spatial resolution.
\end{abstract}

Key words: remote sensing, vegetation index, NOAA, land cover, Southeast Asia

\section{Introduction}

Sound management of the regional, national and global environment requires information acquired by monitoring the earth surface conditions and the changes in these conditions. It is, however, very hard to monitor these conditions and changes continuously over broad areas using ground surveys. Remote sensing from satellites can overcome such difficulties and accomplish the necessary monitoring goals.

Our goal was the creation of mosaiced and cloud-free images with $1 \mathrm{~km}$ spatial resolution from NOAA AVHRR (LAC) data to visualize broad areas of South and South-East Asia including India, Thailand, Malaysia, etc. In addition, a vegetation index map was made from the resulting. images for the purpose of macroscopic monitoring of vegetation conditions and land cover in the region.

\section{Study area and data employed}

The product images cover the area from lat. $0^{\circ}$ to $35^{\circ} \mathrm{N}$ and from long. $66^{\circ}$ to $110^{\circ} \mathrm{E}$ including the whole of India and the Indochinese peninsula.

AVHRR LAC mode data from NOAA-9 and NOAA-11 were used. Images were produced for the years 1986, 1987, 1988, 1989, 1993 and 1994. Since relatively fewer data were available for 1987 , an additional image for $1987 / 1988$ was produced for reference from 
the merged data sets of 1987 and 1988. All data used were acquired in the dry season; that is from December of the previous year to March of the nominal year.

The numbers of original data scenes used for those product images are 18 for 1986, 7 for 1987,15 for 1988, 12 for 1989, 21 for 1993 and 13 for 1994.

\section{Data processing}

\subsection{Data selection}

The LAC computer compatible tapes (CCT) were purchased from the NOAA/National Environmental Satellite, Data, and Information Service. The images were displayed on a monitor and appropriate data sets were selected considering the cloud and area coverage conditions.

\subsection{Radiometric correction}

First, the visible and near-infrared bands (bands 1 and 2) of the original LAC data were converted to albedo as follows:

$$
A_{i}=\left(G_{i} \times D_{i}+O_{i}\right) / \cos \theta, \quad i=1,2
$$

where $A_{i}$ is the albedo in percent, $G_{i}$ and $O_{i}$ are the gain and offset for sensor degradation, respectively, $D_{i}$ is the digital count of the data and $\theta$ is the solar zenith angle. The albedos obtained from equation 1 were converted to eight-bit integer values as follows:

$$
\begin{aligned}
& 0.0 \% \leqq \mathrm{~A}_{\mathrm{i}} \leqq 20.0 \% \quad \mathrm{X}_{\mathrm{i}}=\mathrm{A}_{\mathrm{i}} / 0.2 \\
& 20.0 \% \leqq A_{i} \leqq 50.0 \% \quad X_{i}=\left(A_{i}-20\right) / 0.3+100 \\
& 50.0 \% \leqq A_{i} \leqq 77.5 \% . \quad X_{i}=\left(A_{i}-50\right) / 0.5+200 \\
& 77.5 \% \leqq A_{i} \quad \therefore \quad X_{i}=255
\end{aligned}
$$

The thermal band data, except for those from band 3, were converted to brightness temperature according to the calibration process described in "NOAA POLAR ORBITER DATA USERS GUIDE" (NOAA/NESDIS, 1991) and then converted to eight-bit integers as follows:

$$
X_{i}=\left(T_{j}+50\right) / 0.5, \quad i=3,4 ; j=i+1
$$

where $T_{j}$ is the brightness temperature in band $j\left({ }^{\circ} \mathrm{C}\right)$. The band 3 data were omitted because they were greatly affected by both thermal emissions and solar reflections.

The parameters for the effects of solar illumination and sensor viewing geometry, including solar zenith angle $(\theta)$, satellite zenith angle $(\phi)$ and the relative difference between solar azimuth and satellite azimuth $(\omega)$, were computed for each pixel position. These parameters were converted to eight-bit integers as follows:

$$
\begin{array}{ll}
\mathrm{X}_{5}=\theta / 0.5 & 0^{\circ} \leqq \theta \leqq 90^{\circ} \\
\mathrm{X}_{6}=\phi / 0.5 & 0^{\circ} \leqq \phi \leqq 90^{\circ} \\
\mathrm{X}_{7}=\omega & 0^{\circ} \leqq \omega \leqq 180^{\circ}
\end{array}
$$

The tangent correction of the primary geometric correction, in which the pixel spacing was converted from equiangular to equidistanct intervals, was performed at the same time as the radiometric corrections. 


\subsection{Geometric correction}

The data from the seven bands obtained from the radiometric correction described above were then geometrically corrected. First, a system correction using the tick mark information available in Level 1B LAC-CCT was performed. The corrected data were projected with Lambert's conformal conic projection for which the two standard parallels were $5^{\circ} 50^{\prime} \mathrm{N}$ and $29^{\circ} 10^{\prime} \mathrm{N}$. The pixel size after correction was one square kilometer on the ground. The image size of the final mosaiced product was 5100 pixels by 4000 lines.

\subsection{Mosaicing}

The images after geometric correction were mosaiced into the image product which covered the whole study area. Before mosaicing, the residual geometric distortion was adjusted using ground control points. Cloud elimination for areas where multiple data sets overlapped was also performed during the mosaicing process using the thermal band data and a Normalized Vegetation Index (NVI) derived from the albedos of bands 1 and 2 as follows:

$$
\mathrm{NVI}=(\mathrm{A} 2-\mathrm{A} 1) /(\mathrm{A} 2+\mathrm{A} 1)
$$

In principle, the pixel having the maximum NVI value amongst all pixels for the same location was selected and used for the product image (Fig. 1). Exceptions were made for cases in which the maximum NVI value was smaller than a certain threshold (typically 0.0). For such cases, the pixel having the highest temperature value from AVHRR band 4 was selected to eliminate clouds for areas of desert and water cover. In addition, if the NVI difference amongst the pixels for the same location was smaller than a threshold value (typically 0.1 ), then the pixel having the smallest value in the visible band (AVFRR band 1) was selected. This procedure minimized atmospheric effects.

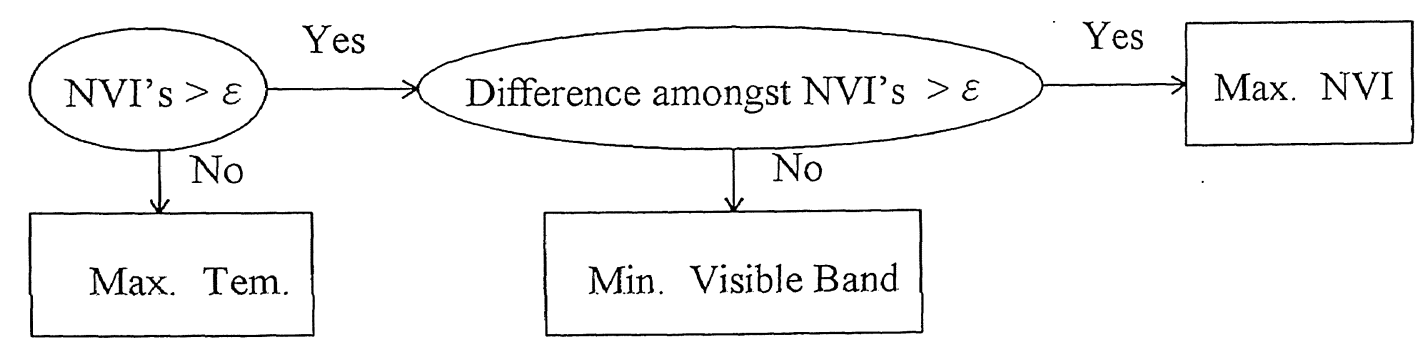

Fig. 1 Flow chart for cloud elimination by overlaying multiple AVHRR data sets.

Following the cloud elimination procedure, the date of observation for each selected pixel was registered in the eighth band of the mosaiced image as the number of days after 30 November except for the 1988 data in the combined 1987/1988 image for which the days were coded as the number of days after 30 November plus 128. The upper panel in Plates 1 is the false color composite of the mosaiced image of the 1994 products (red: band 2, green and blue: band 1).

\subsection{Computation of vegetation index}

The vegetation index was computed using the albedo of AVHRR bands 1 and 2 of 
the mosaiced images. We used the Calibrated Vegetation Index (CVI), defined by NOAA/NESDIS in the "GLOBAL VEGETATION INDEX USERS GUIDE" as follows:

$$
\mathrm{CVI}=260\left(\mathrm{~A}_{2}-\mathrm{A}_{1}\right) /\left(\mathrm{A}_{2}+\mathrm{A}_{1}\right)+15
$$

The lower panel in Plates 1 shows the vegetation index image obtained from the mosaiced image shown in the upper panel.

\subsection{Extraction of land and overlay of national boundary}

The land areas were extracted from the mosaiced images and then the national boundaries were overlaid using the World Boundary Database II in the UNEP/GRID database. Finally the products were exposed directly on 8 -by-10-inch negatives to create the final photographic products.

\section{Concluding remarks}

Mosaiced satellite data and vegetation index images for 1986, 1987, 1988, 1987/1988, 1989, 1993 and 1994 were produced from NOAA AVHRR (LAC) data covering the South and Southeast Asian region with $1 \mathrm{~km}$ spatial resolution. The same kinds of images but for the period from 1990 to 1992 are now being processed and will be available soon.

All the images created by this monitoring project have been archived in the UNEP/GRID database and are available through GRIDTsukuba, losated in the Center for Global Environmental Research (NIES-CGER) which is a part of the National Institute for Environmental Studies.
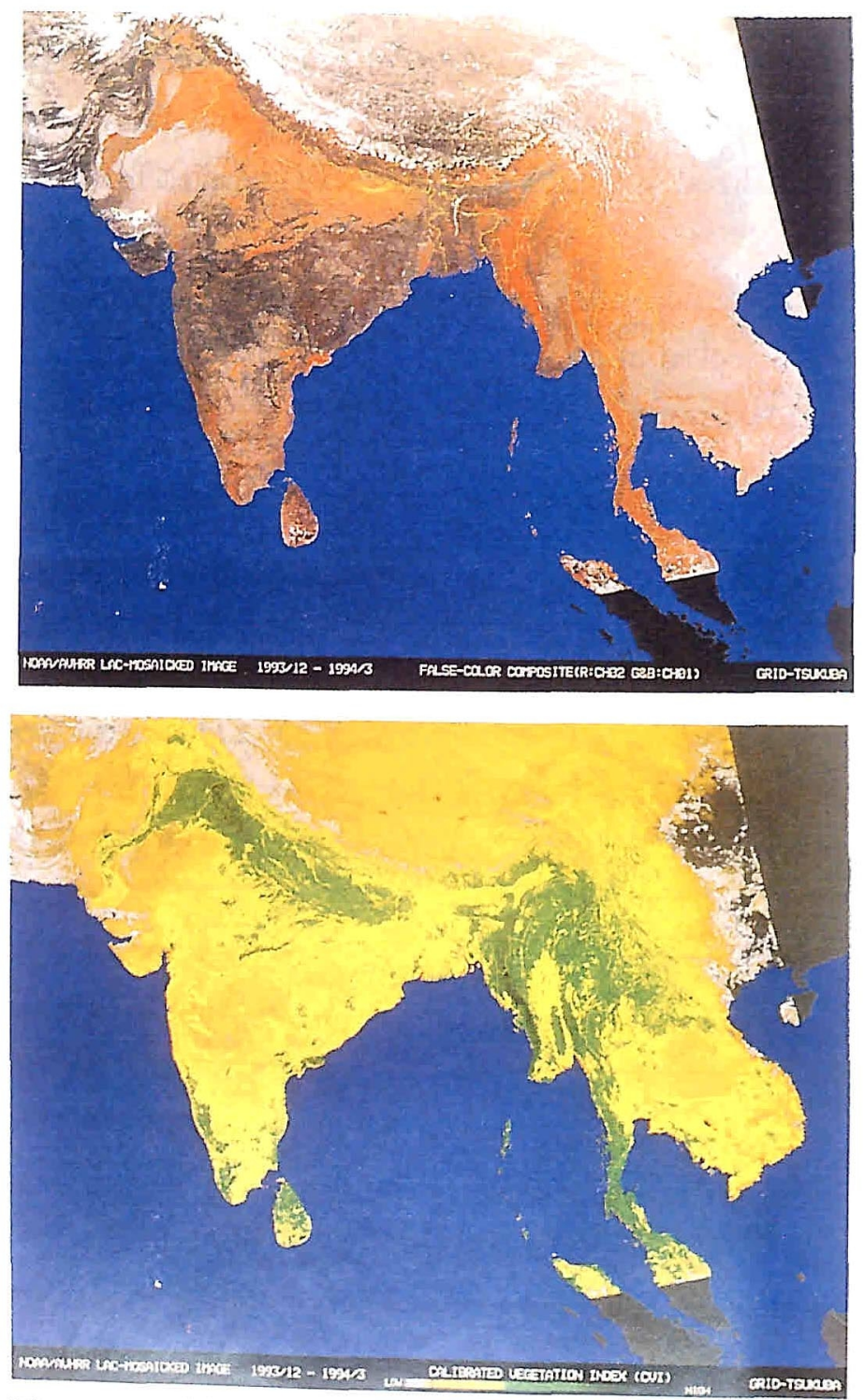

Plate 11994 products.

Upper: False color composite (R: $\mathrm{CH} 2$,

G\&B: CH1) and Lower: Calibrated

Vegetation Index. 